\title{
A study of intracity variation of temperature-related mortality and socioeconomic status among the Chinese population in Hong Kong
}

\author{
Emily Ying Yang Chan, William B Goggins, Jacqueline Jakyoung Kim, Sian M Griffiths
}

- An additional appendix I is published online only. To view this file please visit the journal online (http://jech.bmj.com/ content/66/4.toc)

School of Public Health, The Chinese University of Hong Kong, Prince of Wales Hospital, Shatin, N.T., Hong Kong

\section{Correspondence to} Dr Emily Y Y Chan, 202D, School of Public Health and Primary Care, The Chinese University of Hong Kong, Prince of Wales Hospital, Shatin, N.T., Hong Kong;

emily.chan@cuhk.edu.hk

Accepted 14 August 2010 Published Online First 25 October 2010

\section{ABSTRACT}

Background Hong Kong, a major city in China, has one of the world's highest income inequalities and one of the world's highest average increases in urban ambient temperatures. Heat-related mortality in urban areas may vary with acclimatisation and population characteristics. This study examines how the effect of temperature on mortality is associated with sociodemographic characteristics at an intracity level in Hong Kong, China, during the warm season.

Methods Data from the Hong Kong Observatory, Census and Statistics Department, Environmental Protection Department and government general outpatient clinics during 1998-2006 were used to construct generalised additive (Poisson) models to examine the temperature mortality relationship in Hong Kong. Adjusted for seasonality, long-term trends, pollutants and other potential confounders, effect modification of the warm season temperature-mortality association by demographic, socioeconomic factors and urban design were examined.

Results An average $1^{\circ} \mathrm{C}$ increase in daily mean temperature above $28.2^{\circ} \mathrm{C}$ was associated with an estimated $1.8 \%$ increase in mortality. Heat-related mortality varied with sociodemographic characteristics: women, men less than 75 years old, people living in low socioeconomic districts, those with unknown residence and married people were more vulnerable. Non-cancerrelated causes such as cardiovascular and respiratory infection-related deaths were more sensitive to high temperature effects.

Conclusion Public health protection strategies that target vulnerable population subgroups during periods of elevated temperature should be considered.

Over the past decade, the impact of climate change has led to an increased frequency and intensity of extreme temperatures. The International Panel on Climate Change projects that global climate change through changing weather patterns will result in an increased risk of temperature-related mortality around the world. ${ }^{1}$ Periods of elevated temperature, in particular, are expected to contribute to a greater burden of mortality and morbidity in both high and low income settings. ${ }^{2} 3$

Population sensitivity towards extreme temperature varies with acclimatisation, demographics and socioeconomic characteristics; few studies have examined how intracity socioeconomic inequalities might affect the relation between temperature and mortality. ${ }^{5}$ Moreover, those studies focused on specific causes of heat-related mortality. ${ }^{6}$ A multicountry comparative study found that the pattern of heat-related mortality varied with average city income in urban settings. ${ }^{7}$ In high income cities, older people were more vulnerable to heat-related mortality, whereas in low income cities, mortality tended to accumulate for children. Hong Kong, a major city in China, has one of the world's highest intracity income inequalities and has had one of the world's highest average increase of urban ambient temperature during the past century. ${ }^{8}$ This study aims to: (1) examine the relation between temperature and mortality for all non-accidentalrelated deaths in Hong Kong from 1998 to 2006; (2) explore how the relation between temperature and mortality varies with intracity demographic and socioeconomic status (SES) characteristics; and (3) describe the adverse health outcomes of climate change through temperature during the three hottest years in the past century in Hong Kong (2003-5).

\section{METHODS}

A retrospective ecological study based on routine mortality, temperature and pollution data was conducted. Mortality data were obtained from the Hong Kong Census and Statistics Department and 129688 non-accidental deaths that occurred between May and October from 1998 to 2006 were included in the final analysis. Mean daily temperatures, dew point temperatures and mean humidity were obtained from the Hong Kong Observatory. Pollution levels were obtained from the 11 general environmental collection stations of the Hong Kong Environmental Protection Department. Daily means were calculated from hourly concentrations of nitrogen dioxide, sulphur dioxide, ozone and $\mathrm{PM}_{10}$ (also known as respirable suspended particulates in Hong Kong). ${ }^{9}$ Average weekly consultation rates for influenza-like illnesses reported by general outpatient clinics and general practitioners were obtained from the Centre for Health Promotion to use as a proxy indicator to control for the influence of influenza epidemics on mortality. Median monthly domestic household incomes according to government tertiary planning units (TPU), the smallest geographical unit for which data are released, were obtained as a measure of neighbourhood SES.

The mortality data included age, gender, marital status, reported previous country of residence, area of residence and cause of death. Age was classified as 85 years or over, $75-84$ years and $0-74$ years and marital status as married or non-married. Neighbourhood SES was obtained from the median monthly domestic household income in the TPU of the area of residence, which was categorised as 'SES 
low' (HK\$ $\leq 15000$ per month/US\$23077 per year), 'SES medium' (HK\$15 001-25 000 per month/US\$23 077-38 462 per year) and 'SES high' (HK\$>25000 per month/US\$28 462 per year). Migration status was derived from 'reported previous country of residence' as 'locals', 'migrants from China' and 'other countries'. A measure of urban design was created by grouping 'area of residence' into: (1) Hong Kong Island (less densely populated coastal residence with good ambient air ventilation, younger population); (2) Kowloon (most densely populated area, with urban planning characterised by rows of $80+$ storey buildings that block district ambient air ventilation); (3) New Territories (less densely populated new towns, younger population); and (4) unknown (which included population who are homeless, street sleepers, with transient status, live in area with no official residence district or with unknown residence status) (see supplementary appendix I, available online only, for detailed population density by residential categories). Cause of death was considered as cancer and non-cancer, and further subdivided into 10 subtypes (cardiovascular, respiratory, nephritis, diabetes, septicaemia, aortic aneurism, cirrhosis, respiratory infections, stroke and others).

\section{Statistical model}

Generalised additive (Poisson) models were used to examine the association between daily mean temperature and daily deaths from natural causes during the warm season (May to October). Stata 9 and R 6.2.3 were used for analysis. Confounders considered were air pollution, day of the week and influenza circulation. In several Asian cities, using an average lag of $0-1$ days for pollutants had the strongest effect on mortality. ${ }^{10} 11$ Nitrogen dioxide, sulphur dioxide, respirable suspended particulates and ozone were controlled for by using linear terms for the average of the same day and a lag of 1 day for each pollutant. Day of the week and holidays were controlled for by using indicator variables. Influenza was controlled for using a linear function of the average weekly cases reported to general practitioners and general outpatient clinics. Cubic smoothing spline functions with 40 degrees of freedom (df) were used to control for seasonality and long-term time trends. ${ }^{12}$ With annual data $7 \mathrm{df}$ per year is often used, ${ }^{13}$ but as we only considered 6 months we used $3.5 \mathrm{df}$ per year plus $8 \mathrm{df}$ for bridging the gap between adjacent years. The quasi-Poisson distribution family was used to account for possible data overdispersion. Model residuals were checked for autocorrelation. Sensitivity analysis was performed by varying the degree of freedom for smooth functions of time and temperature for the models.

We originally considered daily temperature as four smooth terms with $4 \mathrm{df}$ each for: (1) same day mean temperature; (2) lag 1 mean temperature; (3) the averages of mean temperatures for lags 2 to 7; and (4) lags 8 to 14. Akaike's information vriterion (AIC) was used to compare models. In the final models, two linear terms were adopted to model the temperature effect on mortality. To account for the heat effect, 'same day mean daily temperature -28.2 for days $>28.2^{\circ} \mathrm{Chapman}$ and 0 for days $\leq 28.2^{\circ} \mathrm{C}^{\prime}$ was used. For modelling the cool effect a linear term, '28.2-average of mean temperature for lags $0-7$ for days with average of mean lag $0-7$ temperature $<28.2^{\circ} \mathrm{C}, 0$ for days for which this average $\geq 28^{\circ} \mathrm{C}^{\prime}$ was used. The threshold temperature was estimated graphically.

\section{RESULTS}

The average daily mean temperature (SD) over the study period was $27.6^{\circ} \mathrm{C}$, with a median of $27.8^{\circ} \mathrm{C}$, a range from $19.7^{\circ} \mathrm{C}$ to $31.8^{\circ} \mathrm{C}$ and an interquartile range from $26.4^{\circ} \mathrm{C}$ to $29.1^{\circ} \mathrm{C}$. During the entire study period, 8 days had a mean temperature below $22.0^{\circ} \mathrm{C}$ (see figure 1 ).

Initially smooth terms for the averages of same day (lag 0), lag 1 , lags $2-7$ and lags 8-14 mean temperatures were examined within the same model simultaneously. After adjustment for confounders, mortality increased consistently with the average

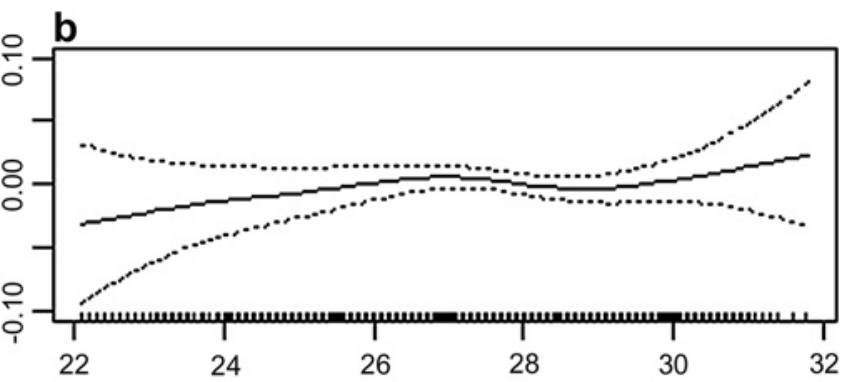

Lag 1 mean temperature

d

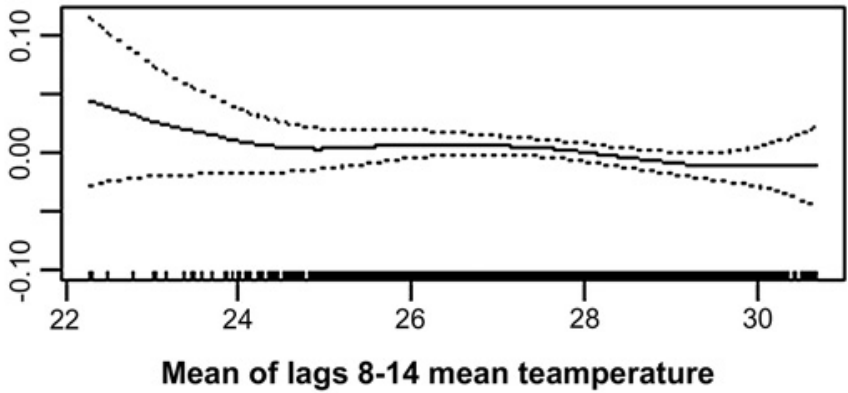

Figure 1 Adjusted smoothed relationships between various lags of mean temperature and the centred log of mortality, all with $4 \mathrm{df}$ and adjusted for seasonality, pollutants, day of week, holidays, influenza rates and the other lagged temperature variables. 
same day mean temperature $(p=0.019)$ (figure $1 \mathrm{~A})$, but there was no clear association with lag 1 mean temperature (figure 1B). A U-shaped association was found for the average lags $2-7$ temperature, with a turning point at approximately $28.2^{\circ} \mathrm{C}$ $(p=0.018)$ (figure 1C), whereas there was no clear association for the average of lags 8-14 mean temperatures (figure 1D). (In the figures, a centered smoothed function of log mortality is shown rather than the actual log of mortality.) Higher daily mean temperatures were associated with increased mortality on the same day and subsequent day over the whole range of temperatures during the warm season, but for temperatures below $28.2^{\circ} \mathrm{C}$ there was a negative association between higher temperatures and mortality 2-7 days later. Subsequent model exploration indicated that the effect of increases in temperature above $28.2^{\circ} \mathrm{C}$ was to raise mortality significantly on the same day but that the lagged effects of high temperatures were very small, so we used a single linear term for same day temperatures over $28.2^{\circ} \mathrm{C}$. For temperatures between $22.0^{\circ} \mathrm{C}$ and $28.2^{\circ} \mathrm{C}$, a linear term 'the mean temperature of lags $0-7$ ' was used to characterise the net short-term effect, as an increase in mortality on the same day appeared to be offset by decreases in mortality over the next few days.

In sensitivity analysis, varying the degrees of freedom from 25 to 60 of the smooth time-related function resulted only in minimal change. Varying the degrees of freedom for temperature (from 2 to 6) also did not affect the basic shape of the smooth function designed to capture temperature effects. Of the four pollutants, sulphur dioxide was the strongest predictor of mortality and the strongest confounder of the temperature pollution association. Models seemed to be insensitive to changes in the number of pollutants controlled for as long as sulphur dioxide was included.

Table 1 shows the RR of temperature-related mortality per $3^{\circ} \mathrm{C}$ increases and decreases in a single day's average daily temperature above $28.2^{\circ} \mathrm{C}$ for Hong Kong 1998-2006. On average, a $1.0^{\circ} \mathrm{C}$ increase in mean temperature above $28.2^{\circ} \mathrm{C}$ for a particular day was associated with an estimated $1.8 \%$ increase in mortality. Deaths among women (RR 1.068, 95\% CI 1.017 to 1.121, $\mathrm{p}=0.008)$ were more sensitive to higher temperatures than among men (RR 1.045, 95\% CI 1.001 to 1.092, $\mathrm{p}=0.046)$. Age was collapsed into two groups $(0-74$ and $\geq 75$ years) because estimates for the 75-84 and over 85 years age groups were almost identical. After stratification we noted that among those less than 75 years old there was little difference between men and womens in terms of the sensitivity of daily mortality to higher temperature (RR (men) 1.067) versus ( $R R$ (women) 1.061 ) but a much larger gender difference in sensitivity for those aged 75 years or older (RR (men) 1.014) versus (RR (women) 1.076).

Temperature-related mortality was negatively associated with SES, with the strongest effects for those living in low SES areas, a weaker effect for those in medium SES areas, and no apparent effect for those in high SES areas. Urban design had little effect on the temperature-mortality relationship but those with unknown residence ( $R R$ 1.278, 95\% CI 1.058 to $1.545, p=0.011$ ) were more vulnerable to heat effects, as were married people.

Stratification by cause of death showed that cancer deaths were insensitive to temperature changes while non-cancer deaths (RR 1.086, 95\% CI 1.041 to 1.132 , p<0.001) were associated with elevated temperature. Table 2 shows RR of causespecific mortality corresponding to $3^{\circ} \mathrm{C}$ increases and decreases in a single day's average daily temperature above $28.2^{\circ} \mathrm{C}$ for Hong Kong 1998-2006. Deaths from cardiovascular diseases (RR $1.085,95 \%$ CI 1.014 to 1.162 ) and respiratory infections (RR
1.102, 95\% CI 0.997 to $1.217, \mathrm{p}=0.053$ ) were sensitive to elevated temperature above $28.2^{\circ} \mathrm{C}$. However, there was a different pattern of temperature mortality for decreasing mean temperature below $28.2^{\circ} \mathrm{C}$ during the warm season. Whereas migrant status and SES status were not significant effect modifiers, deaths among men (RR $1.039,95 \%$ CI 0.995 to 1.085 , $p=0.021$ ), those aged 75 years and older (RR $1.040,95 \%$ CI 1.007 to $1.075 \mathrm{p}=0.019$ ), those with unknown residence ( $R R$ 1.313, 95\% CI 1.129 to $1.528, p=0.000$ ), marital status (RR 1.036, 95\% CI 1.001 to $1.072, p=0.043)$ and non-cancer-related causes (RR $1.034,95 \%$ CI 1.104 to $0.968, p=0.024$ ) were more sensitive to drops in temperature.

\section{DISCUSSION}

We found $28.2^{\circ} \mathrm{C}$ to be a critical threshold for elevated nonaccidental mortality during the warm season in Hong Kong-a city that has had one of the highest income inequalities globally and the fastest increase of ambient urban temperature within the past century. ${ }^{8}$ The temperature-mortality threshold we found is slightly higher than that found for stroke death using maximum temperature in Hong Kong $^{14}$ and higher than the threshold found for European cities. ${ }^{15}$ Nevertheless, our findings are consistent with other Asia-specific studies ${ }^{3} 16-19$ that have examined the effect of temperature on mortality. Our heat mortality threshold of $28.2^{\circ} \mathrm{C}$ is similar to that found for other Asian cities ${ }^{3}$ such as Bangkok $\left(29^{\circ} \mathrm{C}\right)$, Chiang Mai $\left(28^{\circ} \mathrm{C}\right)$ and Delhi, India $\left(29^{\circ} \mathrm{C}\right)$. However, the percentage increase in daily mortality for a $1^{\circ} \mathrm{C}$ increase above these thresholds was higher in those Asian cities (range from $2.4 \%$ to $5.8 \%$ ) than in Hong Kong (1.8\%), most likely due to better infrastructure development and greater access to air conditioning in Hong Kong. ${ }^{20}$ Indeed, a study in Shanghai, China, hypothesised that the increased use of air conditioning might have contributed to the lower heat wave-related mortality in 2003 compared with $1998 .{ }^{17}$

On the other hand, a different pattern was found for increases in temperature between $22.0^{\circ} \mathrm{C}$ and $28.2^{\circ} \mathrm{C}$, which were associated with higher mortality on the same day but lower mortality 2-7 days later. This mortality displacement could be due to 'harvesting', in which mortality is concentrated on the day when elevated temperature occurs and is followed by a subsequent decline because very vulnerable individuals have been removed from the population. Another possible explanation for the temperature-mortality association difference above and below $28.2^{\circ} \mathrm{C}$ may be related to the potential longer-term cold effects that may have offset the short-term heat effects at temperatures lower than $28.2^{\circ} \mathrm{C}$.

Our study indicates that some groups may be more vulnerable to heat-related mortality within the urban community; in particular, those with lower SES, perhaps due to chronic health problems or to their inability either to afford air conditioners or their greater reluctance to use air conditioners due to the electricity costs. As such specific health protection strategies may be necessary to protect vulnerable groups from the adverse health impact of elevated temperature. In addition, similar to other studies, we found that some cardiovascular and respiratory deaths were more sensitive to daily temperature fluctuations. $^{21-23}$ Our findings also indicate that heat might affect several other non-cancer causes of death.

The strengths of this study include comprehensive nonaccidental mortality data and neighbourhood income data from official information sources. The warm season analysis, however, may be a limitation as it only considers heat-related mortality between March and October. Other limitations of this 
Table 1 RR of mortality corresponding to $3^{\circ} \mathrm{C}$ increases and decreases in a single day's average daily temperature above $28.2^{\circ} \mathrm{C}$ for Hong Kong $1998-2006$

\begin{tabular}{|c|c|c|c|c|c|}
\hline \multirow[b]{2}{*}{ Variables } & \multicolumn{2}{|c|}{$3.0^{\circ} \mathrm{C}$ increase above $28.2^{\circ} \mathrm{C}$} & \multicolumn{2}{|c|}{$3.0^{\circ} \mathrm{C}$ decrease below $28.2^{\circ} \mathrm{C}$} & \multirow[b]{2}{*}{$\mathbf{N} \dagger$} \\
\hline & RR (95\% Cl) & p Value & RR (95\% Cl) & $\bar{p}$ Value & \\
\hline All & 1.055 (1.022 to 1.090$)$ & 0.001 & 1.021 (0.997 to 1.046$)$ & 0.022 & 129688 \\
\hline \multicolumn{6}{|c|}{ Previous residence $\neq$ (proxy for migration status) } \\
\hline China & $1.050(1.012$ to 1.090$)$ & 0.010 & $1.022(0.994$ to 1.051$)$ & 0.127 & 99195 \\
\hline \multicolumn{6}{|l|}{ Gender } \\
\hline Male & 1.045 (1.001 to 1.092$)$ & 0.046 & $1.039(0.995$ to 1.085$)$ & 0.021 & 72148 \\
\hline \multicolumn{6}{|l|}{ Age, years } \\
\hline$\geq 75$ & 1.047 (1.002 to 1.094$)$ & 0.043 & 1.040 (1.007 to 1.075$)$ & 0.019 & 70466 \\
\hline$<75$ & 1.065 (1.015 to 1.118$)$ & 0.011 & $0.998(0.963$ to 1.035$)$ & 0.928 & 52793 \\
\hline \multicolumn{6}{|c|}{ Gender and age, years } \\
\hline Male $\geq 75$ & $1.014(0.951$ to 1.082$)$ & 0.666 & $1.058(1.008$ to 1.110$)$ & 0.023 & 33254 \\
\hline Male $<75$ & $1.067(1.005$ to 1.133$)$ & 0.034 & $1.023(0.979$ to 1.070$)$ & 0.308 & 38546 \\
\hline \multicolumn{6}{|c|}{ SES of residential area* } \\
\hline Medium & $1.038(0.989$ to 1.089$)$ & 0.129 & $1.029(0.991$ to 1.067$)$ & 0.135 & 68952 \\
\hline High & $1.003(0.904$ to 1.112$)$ & 0.961 & 1.015 (0.938 to 1.099$)$ & 0.708 & 15080 \\
\hline \multicolumn{6}{|c|}{ Area of residence (proxy for urban design) } \\
\hline Hong Kong & $1.043(0.964$ to 1.129$)$ & 0.296 & $1.022(0.962$ to 1.085$)$ & 0.489 & 25954 \\
\hline Kowloon & $1.041(0.981$ to 1.104$)$ & 0.183 & $1.004(0.960$ to 1.051$)$ & 0.852 & 46974 \\
\hline New Territories & $1.042(0.984$ to 1.103$)$ & 0.158 & $1.015(0.972$ to 1.061$)$ & 0.496 & 49172 \\
\hline Unknown & 1.278 (1.058 to 1.545$)$ & 0.011 & $1.313(1.129$ to 1.528$)$ & 0.000 & 7261 \\
\hline \multicolumn{6}{|l|}{ Marital status } \\
\hline Married & 1.055 (1.007 to 1.105$)$ & 0.023 & $1.036(1.001$ to 1.072$)$ & 0.043 & 64816 \\
\hline Unmarried & 1.042 (0.989 to 1.099$)$ & 0.121 & $1.020(0.981$ to 1.061$)$ & 0.319 & 50185 \\
\hline \multicolumn{6}{|l|}{ Cause of death } \\
\hline Cancer & $1.010(0.959$ to 1.064$)$ & 0.715 & $1.002(0.964$ to 1.041$)$ & 0.938 & 50619 \\
\hline
\end{tabular}

*The proxy socioeconomic status (SES) variable was created first by matching individual'area of residence' within the mortality data to the median monthly domestic household income within that area of residence as reported by government tertiary planning units (the smallest geographical unit for which data are available). Resulted proxy SES was then categorised into SES low (HK $\$ \leq 15000$ per month/US\$23 077 per year), SES medium (HK\$15001-25000 per month/US\$23 077-38 462 per year) and SES high (HK\$>25000 per month/US\$28 462 per year).

$+\mathrm{N}=129688$ non-accidental deaths were included in the analysis. Subgroup totals may not add up due to other or unknown cases.

$\ddagger$ Not shown in this table: reported previous residence from other countries. Information available upon request.

study include the ecological fallacy, as temperature, pollutant exposures and SES were all measured at aggregate levels and the observed associations may not necessarily represent associations existing at an individual level. ${ }^{24}$ Therefore the temperature mortality relationship found may not apply to certain subgroups such as those living in the small proportion of households without air conditioning. Another limitation is the inability to control completely for confounding when evaluating subgroup effect modification because age, SES, gender, cause of death and marital status are all associated with one another and we cannot

Table 2 Subgroup RR of non-cancer-related mortality corresponding to $3^{\circ} \mathrm{C}$ increases and decrease in a single day's average daily temperature above $28.2^{\circ} \mathrm{C}$ for Hong Kong 1998-2006

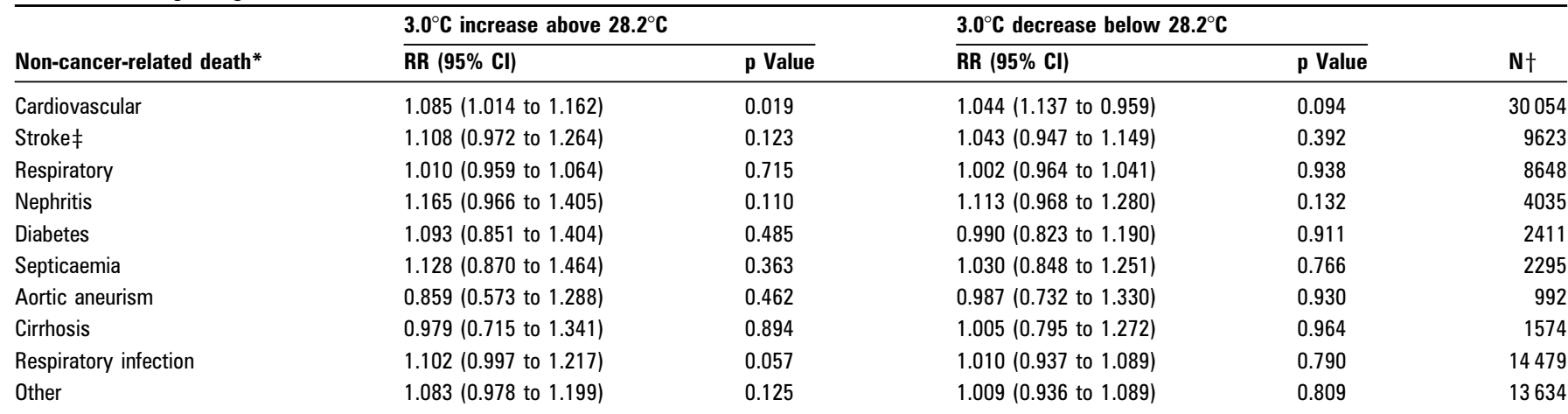

*ICD 9/ICD 10 codes for the studied medical conditions: cancer (ICD9 120-208 or ICD10 C00-C97); cardiovascular (ICD9 390-459 or ICD 10 I00-I99); stroke (ICD9 430-438 or ICD10 I60-I69); respiratory (ICD9 470-478, 490-589 or ICD10 J30-J98); nephritis (ICD9 580-589 or ICD10 N00-N19); diabetes (ICD9 250 or ICD10 E10-E14); septicaemia (ICD9 038 or ICD10 A40-A41); aortic aneurism (ICD9 441 or ICD10 I71); cirrhosis (ICD9 571 or ICD10 K70, K73, K74); respiratory infection (ICD9 460-466, 480-487, 381-382 or ICD10 J00-J06, J10-J18, $\mathrm{H} 65-\mathrm{H} 66, \mathrm{~J} 2 \mathrm{O}-\mathrm{J} 22)$.

$\dagger \mathrm{N}=79069$ total non-cancer deaths were included in the analysis. Subgroup totals may not add up due to other or unknown cases.

$\ddagger$ Stroke (cerebrovascular disease) is a subgroup of cardiovascular. 
stratify by all of these variables simultaneously. In addition, we did not formally assess whether differences by demographic or socioeconomic factors were due to factors other than chance. Moreover, recent urban planning and projects may have impacted temperature variation in different areas of Hong Kong. ${ }^{25}$ In particular, these urban construction projects may have affected Kowloon, where rapidly developing real estate projects have not considered environmental concerns such as urban ambient air ventilation for old buildings in the surrounding areas.

In addition, while we acknowledge that adjusting potential confounding variables will be important to identify the true relationship between study variables and outcomes, our study design and the retrospective databases have limited the possibility of further adjustment for individual level variables because we are using an aggregate outcome, number of deaths and aggregate predictors. We can only directly adjust for aggregate predictors such as pollutant levels and day of the week. The RR in table 1 are not mutually adjusted, ie, they are not adjusted for individual variables such as age, gender and SES.

The analyses stratified by area SES are not adjusted for differences in age distribution, and thus the differences observed between those living in areas with different SES levels could possibly be due to other differences between these areas such as different age distributions, ie, confounding of the SES results by age. Yet, we believe the SES results for high temperature effects are not strongly confounded by differences in age distributions between the low, medium and high SES areas. We did not observe a large difference between age groups in terms of sensitivity of the number of daily deaths due to high temperature (2nd column in table 1) in lower SES areas in Hong Kong (which generally have a larger percentage of residents who are older). We did not observe a large difference between age groups in terms of sensitivity of the number of daily deaths due to high temperature (2nd column in table 1) in lower SES areas in Hong Kong (which generally have a larger percentage of residents who are older)

Further prospective investigation and targeted public protection strategies ${ }^{26-34}$ should be developed. Strategies such as the establishment of heat health warning systems, public service announcements (eg, mass media messages, distribution of educational materials, automated notification systems), cooling centres, information phone lines, hospital alerts (for emergency preparedness), outreach to vulnerable groups (eg, homeless), distribution of cooling methods (eg, fans or air conditioner donations) and environmental projects (eg, urban planning) might be helpful to protect the general public from the adverse impact of elevated temperature. However, the cost-effectiveness

\section{What is already known on this subject}

- The adverse impact of elevated temperature on human mortality varies with acclimatisation, demographics and socioeconomic characteristics.

- Socioeconomic and demographic risk factors for temperaturerelated mortality have been identified; however, there has been no study of the intracity variation in Asian metropolitan areas and the effect of urban design.

- Hong Kong, a major city in China, has one of the world's highest city income inequalities and one of the world's highest average increases of urban ambient temperatures during the past century.

\section{What this study adds}

This is the first study in an urban Chinese city to examine daily mean temperature mortality and its associated socioeconomic effect modifiers. The temperature-mortality threshold in Hong Kong was $28.2^{\circ} \mathrm{C}$ and the average per $1^{\circ} \mathrm{C}$ temperature increase was associated with a $1.8 \%$ increase in mortality above this threshold.

- Subgroup analyses showed that mortality among women, those with unknown residence and married individuals was more sensitive to high temperature effects. An inverse gradient relationship was observed between area level SES and heat-related mortality. Non-cancer-related causes such as cardiovascular and respiratory infection-related death were significantly associated with temperature-related morality in urban Hong Kong, China. While area of residence (a proxy for urban design) was not found to be significantly associated with heat-related mortality, further study might be necessary as the current model cannot ascertain its true impact due to design limitations.

- Epidemiological patterns of mortality in elevated temperature highlight vulnerable population subgroups in urban settings who may need targeted health protection strategies to protect their wellbeing.

of these strategies and the receptiveness of different population subgroups towards these strategies would need to be evaluated.

\section{CONCLUSION}

In Hong Kong, a major city in China with one of the world's highest income inequalities and the highest average increase of urban ambient temperatures during the past century, an average $1^{\circ} \mathrm{C}$ increase in daily mean temperature above $28.2^{\circ} \mathrm{C}$ was associated with an estimated $1.8 \%$ increase in mortality. Some population subgroups were more vulnerable to heat-related mortality, including women, men less than 75 years old, people living in low SES districts, those with unknown residence and married people. Deaths from cardiovascular and respiratory infections were also more sensitive to high temperatures. Targeted health protection strategies should be developed to protect the wellbeing of the population.

Acknowledgement The authors wish to acknowledge the support of Ms Carol Suen, of CUHK SPH, for her support in the preparation of this manuscript.

Funding This work was supported by the Chinese University of Hong Kong, School of Public Health (CUHK SPH) direct grant for research 2006/7 and the Oxford-Li Ka Shing Foundation Collaborative Global Health Research Program. These funding sources serve to provide resources to carry out this research study but have no involvement in study design, data collection, analysis, interpretation, writing of the report or the decision to submit the paper for publication.

\section{Competing interests None.}

Contributors EYYC and WBG were involved in the planning, design, data soliciting, analysis and writing of the report. JJK supported data analysis and report writing. SG was involved in the process of research design and report writing.

Provenance and peer review Not commissioned; externally peer reviewed.

\section{REFERENCES}

1. Intergovernmental Panel on Climate Change. IPCC Fourth Assessment Report: Climate Change 2007(Online). 2007. http://www.ipcc.ch/ipccreports/ar4-wg2.htm. (accessed Jun 22 2008).

2. Pirard $\mathbf{P}$, Vandentorren $\mathrm{S}$, Pascal $\mathrm{M}$, et al. Summary of the mortality impact assessment of the 2003 heat wave in France. Euro Surveill 2005;10:153-6. 
3. McMichael AJ, Wilkinson P, Kovat SJ, et al. International study of temperature, heat and urban mortality: the ISOTHURM project. Int J Epidemiol 2008;37:1-11.

4. Braga ALF, Zanobetti A, Schwartz J. The time course of weather related deaths. Epidemiology 2001;12:662-7.

5. Curriero FC, Heiner KS, Samet JM, et al. Temperature and mortality in 11 cities of the eastern United States. Am J Epidemiol 2003;155:80-7.

6. Gouveia N, Hajat S, Armstrong B. Socioeconomic differentials in the temperature-mortality relationship in São Paulo, Brazil. Int J Epidemiol 2003:32:390-7.

7. Hajat S, Armstrong BG, Gouveia N, et al. Mortality displacement of heat-related deaths: a comparison of Delhi, São Paulo, and London. Epidemiology 2005;16:613-20.

8. United Nations Development Programme. Human Development Report 2006, UNDP Beyond scarcity: Power, poverty and the global water crisis [Online]. 2006. http://hdr.undp.org/en/media/hdr06-complete.pdf (accessed June 13 2008).

9. Environmental Protection Department. The Government of the Hong Kong Special Administrative Region. Definition for Respirable Suspended Particles (RSP) [Online]. 2009. http://www.epd.gov.hk/epd/english/environmentinhk/air/data/ emission inve rsp.html (accessed May 1 2009)

10. Wong CM, Vichit-Vadakan N, Kan H, et al. Public Health and Air Pollution in Asia (PAPA): a multicity study of short-term effects of air pollution on mortality. Environ Health Perspect 2008;116:1195-202.

11. Oian Z, He $\mathrm{O}$, Lin HM, et al. High temperatures enhanced acute mortality effects of ambient particle pollution in the "oven" city of Wuhan, China. Environ Health Perspect 2008:116:1172-8.

12. Wood SN. Generalized additive models: an introduction with $R$. Journal of the Royal Statistical Society: Series A (Statistics in Society): Chapman and Hall, 2006.

13. Roberts S, Martin MA. Applying a moving total mortality count to the cities in the NMMAPS database to estimate the mortality effects of particulate matter air pollution. Occup Environ Med 2006;63:193-7.

14. Leung YK, Yip KM, Yeung KH. Relationship between thermal index and mortality in Hong Kong. Meterol App/ 2008;15:399-409.

15. Hajat S, Armstrong B, Baccini $M$, et al. Impact of high temperatures on mortality: is there an added heat wave effect? Epidemiology 2006:17:632-8.

16. Tan J, Zheng Y, Song G, et al. Heat wave impacts on mortality in Shanghai, 1998 and 2003. Int J Biometeorol 2007:51:193-200.

17. Kan HD, Jia J, Chen BH. Temperature and daily mortality in Shanghai: a time-series study. Biomed Environ Sci 2003;16:133-9.

18. Kan $\mathbf{H}$, London $\mathrm{SJ}$, Chen $\mathrm{H}$, et al. Diurnal temperature range and daily mortality in Shanghai, China. Environ Res 2007:103:424-31.

19. Kim H, Ha JS, Park J. High temperature, heat index, and mortality in 6 major cities in South Korea. Arch Environ Occup Health 2006:61:265-70.
20. Electric and Mechanical Services Department, the Government of Hong Kong Special Administrative Region. Hong Kong Energy End-use Data 2007 (Online). 2007. http://www.emsd.gov.hk/emsd/eng/pee/edata.shtml (accessed Jul 10 2008).

21. Braga AL, Zanobetti A, Schwartz J. The effect of weather on respiratory and cardiovascular deaths in 12 U.S. cities. Environ Health Perspect 2002;110:859-63.

22. Hajat S, Kovats RS, Lachowycz K. Heat-related and cold-related deaths in England and Wales: who is at risk? Occup Environ Med 2007;64:93-100.

23. Saez M, Sunyer J, Castellsagué J, et al. Relationship between weather temperature and mortality: a time series analysis approach in Barcelona. Int J Epidemiol 1995;24:576-82.

24. Freedman DA. Ecological Inference and the Ecological Fallacy. Technical Report [Online]. 1999 No. 549: 1-7. http://www.stanford.edu/class/ed260/freedman549. pdf (accessed Jun 26 2008).

25. Gorer P, Lawson A, Loh C, et al. "Green" House or Greenhouse? Climate Change and the building stock of Hong Kong and Macau. Civic Exchange [Online]. 2008. http://www.civic-exchange.org/eng/upload/files/200804 greenhouse.pdf (accessed Aug 25 2008).

26. Koppe C, Kovats RS, Jendritzky G, et al. Heat-waves: impacts and responses. In: Health and Global Environmental Change Series, No. 2. Copenhagen: WHO Regional Office for Europe, 2004.

27. Sheridan S. A survey of public perception and response to heat warnings across four North American cities: an evaluation of municipal effectiveness. Int $J$ Biometeorol 2007:52:3-15.

28. Smoyer-Tomic KE, Rainham DG. Beating the heat: development and evaluation of a Canadian hot weather health-response plan. Environ Health Perspect 2001;109:1241-8.

29. Nicholls N, Skinner C, Loughnan M, et al. A simple heat alert system for Melbourne Australia. Int J Biometeorol 2008;52:375-84.

30. Pascal M, Laaidi K, Ledrans $\mathbf{M}$, et al. France's heat health watch warning system Int J Biometeorol 2006:50:144-53.

31. Bassil K, Cole D, Smoyer-Tomic K, et al. What is the evidence on applicability and effectiveness of public health interventions in reducing morbidity and mortality during heat episodes? A review for the National Collaborating Centre for Environmental Health [Online]. 2007. http://www.ncceh.ca/files/Heat_Interventions_Apr_2007.pdf (accessed 14 Aug 2009).

32. Woodruff RE, McMichael T, Butler $\mathrm{C}$, et al. Action on climate change: the health risks of procrastinating. Aust N Z J Public Health 2006;30:567-71.

33. Matthies F, Menne B. Prevention and management of health hazards related to heatwaves. Int J Circumpolar Health 2009;68:8-22.

34. Bernard SM, McGeehin MA. Municipal heat wave response plans. Am J Public Health 2004:94:1520-2. 